\title{
The Use of Online Tools in Microscopy and Microanalysis Core Facilities.
}

\author{
Miles Apperley ${ }^{1}$, Jenny Whiting ${ }^{1}$, Bronwen $\mathrm{Cribb}^{2}$ and Anna Ceguerra ${ }^{3}$
}

1. Australian Microscopy and Microanalysis Research Facility, Madsen Building F09, The University of Sydney, NSW, 2006, Australia.

2. Center for Microscopy and Microanalysis, The University of Queensland, Brisbane, QLD, 4072, Australia

3. Australian Center for Microscopy and Microanalysis and the School of Aerospace, Mechanical and Mechatronic Engineering, Madsen Building F09, The University of Sydney, NSW, 2006, Australia.

The Australian Microscopy and Microanalysis Research Facility (AMMRF) is a national grid of equipment, instrumentation and expertise in microscopy and microanalysis that provides nanostructural characterisation capability and services, from widely used optical, electron, X-ray and ion-beam techniques to world-leading flagship platforms. This collaborative facility, comprising a distributed network of microscopy and microanalysis core facilities spread over fourteen institutions, manages a fully supported user experience to more than 3,000 researchers annually.

Once a researcher has identified the facility thy need to work with, each core facility is responsible for supporting the users through a common research experience. This experience or work flow comprises the stages of defining the scientific question and project formulation, identifying techniques, registering a project and a meeting to discuss the project, plan training and arrange access to instrumentation. Later stages include data acquisition and analysis, data management and ultimately an outcome such as a research publication, grant application or invention registration or patent. With more than 3,000 users annually there is a strong need to adopt processes that enable users to be productive as well as to enable efficient management by facility staff at all stages of the research experience.

The Technique Finder (TF) is a web application that enables prospective facility users to identify the techniques most suited to their research, based on a researcher-centric approach and terminology as opposed to instrument-oriented jargon. Specifically, it offers two areas, one for biological scientists and another for researchers in physical sciences, which allow them to identify techniques based on research dimensions in corresponding fields. In addition, it offers a term search based on a comprehensive term index created for each technique including all the directly and indirectly linked information available in the application. The techniques themselves display a full description with sample examples, key reviews and potential for case studies and links. Locations and contact details to each of the AMMRF facilities invite users to get started immediately.

To meet the training needs of microscopy core facilities, the AMMRF has developed MyScope: Training for Advanced Research [2]. MyScope is an online suite of education tools for teaching and learning in the area of microscopy and microanalysis. It comprises a range of modules including scanning electron microscopy; transmission electron microscopy; scanning probe and atomic force microscopy; microanalysis; confocal microscopy; and X-ray diffraction techniques. The modules provide a novel advancement in online training. They contain a number of components including: an interactive questionnaire to allow the user to assess their knowledge, guide choices and tailor the learning environment for flexible learning; also, tailoring capability for academics and trainers; self guided tutorials with videos, animations and glossary to prepare students with knowledge and specialist 
language; virtual instrument platforms to practice use of instrumentation; and online competency testing to demonstrate readiness for hands-on experience [3]. Global interest in MyScope since it was launched in 2011 has grown markedly with more than 150,000 visits from over 20 countries in 2014.

The Characterisaton Virtual Laboratory (CVL) is a cloud-based data analysis and visualisation platform that integrates existing analysis tools and techniques with a network of specialised cloud-based computing systems and data-storage facilities. Currently the CVL hosts tools for analysis of data from cryo-EM, X-ray microCT and atom probe platforms. Specifically the Atom Probe Workbench (APW) enables that enables the atom probe research community to access and create valuable tools, accelerating the research process. Features of the workbench includes an atom probe data management system that performs automatic ingestion of data \& meta-data directly from instruments, processing-modules for accessing reconstruction and analysis techniques, a visualisation engine for exploring data and support for the preparation of publication-quality images .

\section{References:}

[1] S P Ringer and M H Apperley, Networking strategies of the microscopy community for improved utilization of advanced instruments: (1) The Australian Microscopy and Microanalysis Research Facility (AMMRF), C. R. Physique 15 (2014) 269-275.

[2] B Cribb et al, Advanced microscopic characterisation through integrated learning tools, Microscopy and Microanalysis, Volume 17, Issue S2, July 2011, pp 870 - 871

[3] B Cribb et al, MyScope: a national approach to education in advanced microscopic characterization through integrated learning tools, Office for Learning and Teaching, Australian Government Department of Education, ISBN: 978-1-925092-03-5 